\title{
Symmetries of some classes of dynamical systems
}

Cristian Lăzureanu, Tudor Bînzar

To cite this article: Cristian Lăzureanu, Tudor Bînzar (2015) Symmetries of some classes of dynamical systems, Journal of Nonlinear Mathematical Physics 22:2, 265-274, DOI: https://doi.org/10.1080/14029251.2015.1033237

To link to this article: https://doi.org/10.1080/14029251.2015.1033237

Published online: 04 January 2021 


\title{
Symmetries of some classes of dynamical systems
}

\author{
Cristian Lăzureanu \\ Department of Mathematics, Politehnica University of Timişoara, Piaţa Victoriei nr. 2 \\ Timişoara, 300006, România \\ cristian.lazureanu@upt.ro \\ Tudor Bînzar \\ Department of Mathematics, Politehnica University of Timişoara, Piaţa Victoriei nr. 2 \\ Timişoara, 300006, România \\ tudor.binzar@upt.ro
}

Received 11 November 2014

Accepted 27 February 2015

\begin{abstract}
In this paper a three-dimensional system with five parameters is considered. For some particular values of these parameters, one finds known dynamical systems. The purpose of this work is to study some symmetries of the considered system, such as Lie-point symmetries, conformal symmetries, master symmetries and variational symmetries. In order to present these symmetries we give constants of motion. Using Lie group theory, Hamiltonian and bi-Hamiltonian structures are given. Also, symplectic realizations of Hamiltonian structures are presented. We have generalized some known results and we have established other new results. Our unitary presentation allows the study of these classes of dynamical systems from other points of view, e.g. stability problems, existence of periodic orbits, homoclinic and heteroclinic orbits.
\end{abstract}

Keywords: symmetries, symplectic realization, Lie groups, Poisson structure, Hamiltonian dynamics, Lagrangian dynamics.

\section{Introduction}

The importance of the notion of symmetry for ordinary and partial differential equations is emphasized especially in time-evolution problems, bifurcation theory, fluid dynamics. A symmetry group of a system of differential equations is a Lie group which allows us to find some solutions invariant under some of its subgroups.

The symmetry analysis of differential equations was introduced and developed by Sophus Lie [17]. Emmy Noether pointed the connection between symmetries and the existence of conservation laws [19]. The symmetry approach for partial differential equations can be found for example in [12]. For some systems of differentials equations of even order, master symmetries and symmetries were calculated [6]. A classification of the symmetry group of three-dimensional Hamiltonian systems was given in [10]. Recently, some studies of symmetries for a five-dimensional dynamical system was presented [3].

Theoretical details about symmetries of differential equations can be found in $[2,7,20]$. 
The aim of the present paper is to give a unitary presentation of some symmetries for some classes of three-dimensional dynamical systems. For this purpose we have introduced the system:

$$
\left\{\begin{array}{l}
\dot{x}=a y+b y z \\
\dot{y}=c x z+d x \\
\dot{z}=-k x y
\end{array}\right.
$$

where $a, b, c, d, k$ are real parameters, $k \neq 0$. System (1.1) generalizes for example the Euler equations for a free rigid body [20], the equations of the rigid body with a free spinning rotor [14], the real Maxwell-Bloch equations [11], a particular case of Rikitake system [22], a particular case of Rabinovich system [21], a completely integrable case of the Lorenz system [13], a particular case of May-Leonard system [13] and a particular case of Chen-Lee system [4].

In order to establish some symmetries for system (1.1), Hamilton-Poisson realizations and symplectic realizations are given.

For details on Poisson geometry and Hamiltonian mechanical systems, see, e.g. [5, 16, 18].

\section{Hamiltonian structures and symplectic realizations}

In this section Hamiltonian structures and symplectic realizations for the system (1.1) are given. Also, bi-Hamiltonian formulations of the system are presented. Our study will be focused on the following cases.

Case I. $b d-a c \neq 0$.

It is easy to see that the functions $H_{1}, C_{1} \in C^{\infty}\left(\mathbb{R}^{3}, \mathbb{R}\right)$ where $H_{1}(x, y, z)=-\frac{d}{2} x^{2}+\frac{a}{2} y^{2}+\frac{a c-b d}{2 k} z^{2}$ and $C_{1}(x, y, z)=\frac{c k}{2} x^{2}-\frac{b k}{2} y^{2}+(a c-b d) z$ are constants of motion for the considered system.

We begin our study by giving the Lie-Poisson structures. To do this, we consider the following subcases.

I1. $b c \neq 0$.

Proceeding as in [15], let us consider the linear Poisson bracket $\{\cdot, \cdot\}$,

$$
\{x, y\}=\alpha_{1} x+\alpha_{2} y+\alpha_{3} z,\{x, z\}=\beta_{1} x+\beta_{2} y+\beta_{3} z,\{y, z\}=\gamma_{1} x+\gamma_{2} y+\gamma_{3} z .
$$

Imposing the condition that $C=H_{1}$ to be a Casimir for (2.1), it results $\alpha_{1}=\alpha_{2}=\beta_{1}=\beta_{3}=$ $\gamma_{2}=\gamma_{3}=0$. If $H=C_{1}$ is a Hamiltonian function, it follows $\{x, y\}=-\frac{1}{k} z,\{x, z\}=\frac{a}{a c-b d} y$ and $\{y, z\}=\frac{d}{a c-b d} x$, or in coordinates, using matrix notation,

$$
\Pi(x, y, z)=\left[\begin{array}{ccc}
0 & -\frac{1}{k} z & \frac{a}{a c-b d} y \\
\frac{1}{k} z & 0 & \frac{d}{a c-b d} x \\
-\frac{a}{a c-b d} y & -\frac{d}{a c-b d} x & 0
\end{array}\right] .
$$

Therefore we consider the three-dimensional Lie algebra $\mathfrak{g}_{1}$ defined by $\left[E_{1}, E_{2}\right]=-\frac{1}{k} E_{3},\left[E_{1}, E_{3}\right]=$ $\frac{a}{a c-b d} E_{2},\left[E_{2}, E_{3}\right]=\frac{d}{a c-b d} E_{1}$ where

$$
E_{1}=\left[\begin{array}{ccc}
0 & -\frac{1}{k} & 0 \\
\frac{a}{a c-b d} & 0 & 0 \\
0 & 0 & 0
\end{array}\right], E_{2}=\left[\begin{array}{ccc}
0 & 0 & \frac{1}{k} \\
0 & 0 & 0 \\
\frac{d}{a c-b d} & 0 & 0
\end{array}\right], E_{3}=\left[\begin{array}{ccc}
0 & 0 & 0 \\
0 & 0 & -\frac{a}{a c-b d} \\
0-\frac{d}{a c-b d} & 0
\end{array}\right] .
$$

Now, we introduce a second Poisson structure. 
If $b c>0$, we consider the three-dimensional Lie group $E(1,1)$ of rigid motions of the Minkowski plane and its corresponding Lie algebra $\mathfrak{e}(1,1)$. Considering the base $B_{\mathfrak{e}(1,1)}=$ $\left\{E_{1}, E_{2}, E_{3}\right\}$ where

$$
E_{1}=\left[\begin{array}{lll}
0 & 0 & 0 \\
b & 0 & 0 \\
0 & 0 & 0
\end{array}\right], E_{2}=\left[\begin{array}{ccc}
0 & 0 & 0 \\
0 & 0 & 0 \\
\sqrt{b c} & 0 & 0
\end{array}\right], E_{3}=\left[\begin{array}{ccc}
0 & 0 & 0 \\
0 & 0 & \frac{-k \sqrt{b c}}{a c-b d} \\
0 & \frac{-k \sqrt{b c}}{a c-b d} & 0
\end{array}\right],
$$

the following bracket relations $\left[E_{1}, E_{2}\right]=0,\left[E_{1}, E_{3}\right]=\frac{b k}{a c-b d} E_{2},\left[E_{2}, E_{3}\right]=\frac{c k}{a c-b d} E_{1}$, hold.

Following [16], it is easy to see that the bilinear map $\Theta: \mathfrak{e}(1,1) \times \mathfrak{e}(1,1) \rightarrow \mathbb{R}$ given by the matrix $\left(\Theta_{i j}\right)_{1 \leq i, j \leq 3}, \Theta_{12}=-\Theta_{21}=1$ and 0 otherwise, is a 2-cocycle on $\mathfrak{e}(1,1)$ and it is not a coboundary since $\Theta\left(E_{1}, E_{2}\right)=1 \neq 0=f\left(\left[E_{1}, E_{2}\right]\right)$, for every linear map $f, f: \mathfrak{e}(1,1) \rightarrow \mathbb{R}$. On the dual space $\mathfrak{e}(1,1)^{*} \simeq \mathbb{R}^{3}$, a modified Lie-Poisson structure is given in coordinates by

$$
\Pi_{b}(x, y, z)=\left[\begin{array}{ccc}
0 & 1 & \frac{b k}{a c-b d} y \\
-1 & 0 & \frac{c k}{a c-b d} x \\
-\frac{b k}{a c-b d} y & -\frac{c k}{a c-b d} x & 0
\end{array}\right] .
$$

If $b c<0$, we consider the special Euclidean Lie group $S E(2)$ of all orientation-preserving isometries and its corresponding Lie algebra $\mathfrak{s e}(2)$. Considering the base $B_{\mathfrak{s e}(2)}=\left\{X_{1}, X_{2}, X_{3}\right\}$ where

$$
X_{1}=\left[\begin{array}{lll}
0 & 0 & 0 \\
b & 0 & 0 \\
0 & 0 & 0
\end{array}\right], X_{2}=\left[\begin{array}{ccc}
0 & 0 & 0 \\
0 & 0 & 0 \\
\sqrt{-b c} & 0 & 0
\end{array}\right], X_{3}=\left[\begin{array}{ccc}
0 & 0 & 0 \\
0 & 0 & \frac{k \sqrt{-b c}}{a c-b d} \\
0 & \frac{-k \sqrt{-b c}}{a c-b d} & 0
\end{array}\right],
$$

the following bracket relations $\left[X_{1}, X_{2}\right]=0,\left[X_{1}, X_{3}\right]=\frac{b k}{a c-b d} X_{2},\left[X_{2}, X_{3}\right]=\frac{c k}{a c-b d} X_{1}$, hold. Then, on the dual space $\mathfrak{s e}(2)^{*} \simeq \mathbb{R}^{3}$, the same Lie-Poisson structure $\Pi_{b}$ is obtained.

I2. $b=0$.

In this case, we consider the three-dimensional Heisenberg Lie group $\mathrm{H}_{3}$ and its corresponding Lie algebra $\mathfrak{h}_{3}$. Considering the base $B_{\mathfrak{h}_{3}}=\left\{E_{1}, E_{2}, E_{3}\right\}$ where

$$
E_{1}=\left[\begin{array}{lll}
0 & 0 & a \\
0 & 0 & 0 \\
0 & 0 & 0
\end{array}\right], E_{2}=\left[\begin{array}{lll}
0 & 1 & 0 \\
0 & 0 & 0 \\
0 & 0 & 0
\end{array}\right], E_{3}=\left[\begin{array}{lll}
0 & 0 & 0 \\
0 & 0 & k \\
0 & 0 & 0
\end{array}\right],
$$

the following bracket relations $\left[E_{1}, E_{2}\right]=0,\left[E_{1}, E_{3}\right]=0,\left[E_{2}, E_{3}\right]=\frac{k}{a} E_{1}$, hold. As in the case I1, a modified Lie-Poisson structure on the dual space $\mathfrak{h}_{3}^{*} \simeq \mathbb{R}^{3}$ is given in coordinates by

$$
\Pi_{0}=\left[\begin{array}{ccc}
0 & 1 & 0 \\
-1 & 0 & \frac{k}{a} x \\
0 & -\frac{k}{a} x & 0
\end{array}\right]
$$

We notice that for $b=0$ in the matrix $\Pi_{b}$, we obtain $\Pi_{0}$.

I3. $c=0$.

By using the substitution $x=Y, y=X, z=Z$, we obtain the case $\mathbf{I 2}$.

The following theorem gives a Hamilton-Poisson realization and a bi-Hamiltonian formulation of the considered system. 
Theorem 2.1. Let $a, b, c, d, k \in \mathbb{R}$ such that $b d-a c \neq 0$. The system (1.1) has the HamiltonPoisson realization $\left(\mathbb{R}^{3}, \Pi_{b}, H_{1}\right)$ and $C_{1}$ is a Casimir of this configuration. Moreover, system (1.1) has bi-Hamiltonian formulation.

The next theorem states that the system (1.1) can be regarded as a Hamiltonian mechanical system.

Theorem 2.2. Let $a, b, c, d, k \in \mathbb{R}$ such that $b d-a c \neq 0$. The Hamilton-Poisson mechanical system $\left(\mathbb{R}^{3}, \Pi_{b}, H_{1}\right)$ has a full symplectic realization $\left(\mathbb{R}^{4}, \omega, \tilde{H}_{1}\right)$, where $\omega=d p_{1} \wedge d q_{1}+d p_{2} \wedge d q_{2}$ and $\tilde{H}_{1}=-\frac{d}{2} q_{1}^{2}+\frac{a}{2} p_{1}^{2}-\frac{1}{8 k(b d-a c)}\left(c k q_{1}^{2}-b k p_{1}^{2}-2 p_{2}\right)^{2}$.

Proof. The corresponding Hamilton's equations are

$$
\left\{\begin{array}{l}
\dot{q}_{1}=a p_{1}+\frac{b}{2(b d-a c)} p_{1}\left(c k q_{1}^{2}-b k p_{1}^{2}-2 p_{2}\right) \\
\dot{q}_{2}=\frac{1}{2 k(b d-a c)}\left(c k q_{1}^{2}-b k p_{1}^{2}-2 p_{2}\right) \\
\dot{p}_{1}=d q_{1}+\frac{c}{2(b d-a c)} q_{1}\left(c k q_{1}^{2}-b k p_{1}^{2}-2 p_{2}\right) \\
\dot{p}_{2}=0 .
\end{array}\right.
$$

We define the application $\varphi: \mathbb{R}^{4} \rightarrow \mathbb{R}^{3}$ by $\varphi\left(q_{1}, q_{2}, p_{1}, p_{2}\right)=(x, y, z)$ where $x=q_{1}, y=p_{1}, z=$ $\frac{c k}{2(b d-a c)} q_{1}^{2}-\frac{b k}{2(b d-a c)} p_{1}^{2}-\frac{1}{b d-a c} p_{2}$. It follows that $\varphi$ is a surjective submersion, the equations (2.2) are mapped onto the equations (1.1) and the canonical structure $\{., .\}_{\omega}$ is mapped onto the Poisson structure $\Pi_{b}$, as required. We also remark that $H_{1}=\tilde{H}_{1}$ and $C_{1}=p_{2}$.

Case II. $b d-a c=0$.

We notice that if $b c=0$, the system (1.1) is reduced to the harmonic oscillator. In the following we will only consider the case $b c \neq 0$. We remark that the constants of motion of the system (1.1) are the functions $H_{2}, C_{2} \in C^{\infty}\left(\mathbb{R}^{3}, \mathbb{R}\right)$ where $H_{2}(x, y, z)=\frac{k}{2} x^{2}+\frac{b}{2}\left(z+\frac{a}{b}\right)^{2}$ and $C_{2}(x, y, z)=\frac{c}{2} x^{2}-\frac{b}{2} y^{2}$. In order to obtain a Hamilton-Poisson realization of the system (1.1) we consider two cases.

If $b c>0$, we again consider the Lie algebra $\mathfrak{e}(1,1)$ having now the base $B_{\mathfrak{e}(1,1)}=\left\{E_{1}, E_{2}, E_{3}\right\}$ where

$$
E_{1}=\left[\begin{array}{lll}
0 & 0 & 0 \\
1 & 0 & 0 \\
0 & 0 & 0
\end{array}\right], E_{2}=\left[\begin{array}{ccc}
0 & 0 & 0 \\
0 & 0 & 0 \\
\sqrt{\frac{c}{b}} & 0 & 0
\end{array}\right], E_{3}=\left[\begin{array}{ccc}
0 & 0 & 0 \\
0 & 0 & -\sqrt{\frac{c}{b}} \\
0-\sqrt{\frac{c}{b}} & 0
\end{array}\right]
$$

with $\left[E_{1}, E_{2}\right]=0,\left[E_{1}, E_{3}\right]=E_{2},\left[E_{2}, E_{3}\right]=\frac{c}{b} E_{1}$. On the dual space $\mathfrak{e}(1,1)^{*} \simeq \mathbb{R}^{3}$, a Lie-Poisson structure is given in coordinates by

$$
\widetilde{\Pi}_{1}(x, y, z)=\left[\begin{array}{ccc}
0 & 0 & y \\
0 & 0 & \frac{c}{b} x \\
-y & -\frac{c}{b} x & 0
\end{array}\right]
$$

If $b c<0$, once more we consider the Lie algebra $\mathfrak{s e}(2)$ with the base $B_{\mathfrak{s e}(2)}=\left\{X_{1}, X_{2}, X_{3}\right\}$ where

$$
X_{1}=\left[\begin{array}{lll}
0 & 0 & 0 \\
1 & 0 & 0 \\
0 & 0 & 0
\end{array}\right], X_{2}=\left[\begin{array}{rrr}
0 & 0 & 0 \\
0 & 0 & 0 \\
-\sqrt{-\frac{c}{b}} & 0 & 0
\end{array}\right], X_{3}=\left[\begin{array}{ccc}
0 & 0 & 0 \\
0 & 0 & -\sqrt{-\frac{c}{b}} \\
0 & \sqrt{-\frac{c}{b}} & 0
\end{array}\right]
$$


with $\left[X_{1}, X_{2}\right]=0,\left[X_{1}, X_{3}\right]=X_{2},\left[X_{2}, X_{3}\right]=\frac{c}{b} X_{1}$. Therefore, on the dual space $\mathfrak{s e}(2)^{*} \simeq \mathbb{R}^{3}$, the same Lie-Poisson structure $\widetilde{\Pi}_{1}$ is obtained.

Now, we can state the next result.

Theorem 2.3. Let $a, b, c, d, k \in \mathbb{R}$ such that $b d-a c=0$. The system (1.1) has the Hamilton-Poisson realization $\left(\mathbb{R}^{3}, \widetilde{\Pi}_{1}, H_{2}\right)$ and $C_{2}$ is a Casimir of this configuration.

In the following, another Poisson structure is given. Let us consider the modified Poisson bracket $\{\cdot, \cdot\}[16]$,

$$
\{x, y\}=\alpha_{1} x+\alpha_{2} y+\alpha_{3} z+\alpha_{4},\{x, z\}=\beta_{1} x+\beta_{2} y+\beta_{3} z+\beta_{4},\{y, z\}=\gamma_{1} x+\gamma_{2} y+\gamma_{3} z+\gamma_{4} .
$$

Imposing the condition that $C=H_{2}$ is a Casimir for (2.3), it results $\alpha_{1}=\alpha_{2}=\beta_{1}=\beta_{2}=\beta_{3}=\beta_{4}=$ $\gamma_{2}=\gamma_{3}=\gamma_{4}=0, a \gamma_{1}=k \alpha_{4}, b \gamma_{1}=k \alpha_{3}$. If $H=C_{2}$ is a Hamiltonian function, we get the following dynamical system:

$$
\left\{\begin{array}{l}
\dot{x}=-b \alpha_{4} y-b \alpha_{3} y z \\
\dot{y}=-c \alpha_{3} x z-c \alpha_{4} x \\
\dot{z}=b \gamma_{1} x y
\end{array}\right.
$$

Taking $\alpha_{3}=-1, \alpha_{4}=-\frac{a}{b}, \gamma_{1}=-\frac{k}{b}$, the above system is the system (1.1) in the case $b d-a c=0$ with $b c \neq 0$. Thus, $\{x, y\}=-z-\frac{a}{b},\{x, z\}=0,\{y, z\}=-\frac{k}{b} x$, or in coordinates, using matrix notation,

$$
\widetilde{\Pi}_{2}(x, y, z)=\left[\begin{array}{ccc}
0 & -z-\frac{a}{b} & 0 \\
z+\frac{a}{b} & 0 & -\frac{k}{b} x \\
0 & \frac{k}{b} x & 0
\end{array}\right] .
$$

Therefore we consider the three-dimensional Lie algebra $\mathfrak{g}_{2}$ defined by $\left[Y_{1}, Y_{2}\right]=-Y_{3},\left[Y_{1}, Y_{3}\right]=0$, $\left[Y_{2}, Y_{3}\right]=-\frac{k}{b} Y_{1}$ where

$$
Y_{1}=\left[\begin{array}{lll}
0 & 0 & 0 \\
1 & 0 & 0 \\
0 & 0 & 0
\end{array}\right], Y_{2}=\left[\begin{array}{ccc}
0 & 0 & 0 \\
0 & 0 & -\sqrt{\frac{k}{b}} \\
0 & \sqrt{\frac{k}{b}} & 0
\end{array}\right], Y_{3}=\left[\begin{array}{ccc}
0 & 0 & 0 \\
0 & 0 & 0 \\
\sqrt{\frac{k}{b}} & 0 & 0
\end{array}\right]
$$

and $k b>0$, and respectively $\mathfrak{g}_{3}$ defined by $\left[Z_{1}, Z_{2}\right]=-Z_{3},\left[Z_{1}, Z_{3}\right]=0,\left[Z_{2}, Z_{3}\right]=-\frac{k}{b} Z_{1}$ where

$$
Z_{1}=\left[\begin{array}{lll}
0 & 0 & 0 \\
1 & 0 & 0 \\
0 & 0 & 0
\end{array}\right], Z_{2}=\left[\begin{array}{ccc}
0 & 0 & 0 \\
0 & 0 & \sqrt{-\frac{k}{b}} \\
0 & \sqrt{-\frac{k}{b}} & 0
\end{array}\right], Z_{3}=\left[\begin{array}{ccc}
0 & 0 & 0 \\
0 & 0 & 0 \\
\sqrt{-\frac{k}{b}} & 0 & 0
\end{array}\right]
$$

and $k b<0$.

After standard computations it follows that the Lie group $G_{2}$ generated by the Lie algebra $\mathfrak{g}_{2}$ is $S E(2)$ and the Lie group $G_{3}$ generated by the Lie algebra $\mathfrak{g}_{3}$ is $E(1,1)$.

Now, it is easy to prove the following result.

Theorem 2.4. Let $a, b, c, d, k \in \mathbb{R}$ such that $b d-a c=0$. The system (1.1) is a bi-Hamiltonian system. 
The next theorems state that the system (1.1) can be regarded as a Hamiltonian mechanical system.

Theorem 2.5. Let $a, b, c, d, k \in \mathbb{R}$ such that $b d-a c=0$. If $b c>0$, then the Hamilton-Poisson mechanical system $\left(\mathbb{R}^{3}, \widetilde{\Pi}_{1}, H_{2}\right)$ has a full symplectic realization $\left(\mathbb{R}^{4}, \omega, \tilde{H}_{2}^{+}\right)$where $\omega=d p_{1} \wedge$ $d q_{1}+d p_{2} \wedge d q_{2}$ and $\tilde{H}_{2}^{+}=\frac{k}{8}\left(p_{2} e^{q_{1}}+e^{-q_{1}}\right)^{2}+\frac{c}{2} p_{1}^{2}$.

Proof. The corresponding Hamilton's equations are

$$
\left\{\begin{array}{l}
\dot{q}_{1}=c p_{1} \\
\dot{q}_{2}=\frac{k}{4}\left(p_{2} e^{q_{1}}+e^{-q_{1}}\right) e^{q_{1}} \\
\dot{p}_{1}=-\frac{k}{4}\left(p_{2} e^{q_{1}}+e^{-q_{1}}\right)\left(p_{2} e^{q_{1}}-e^{-q_{1}}\right) \\
\dot{p}_{2}=0 .
\end{array}\right.
$$

We define the application $\varphi: \mathbb{R}^{4} \rightarrow \mathbb{R}^{3}$ by $\varphi\left(q_{1}, q_{2}, p_{1}, p_{2}\right)=(x, y, z)$ where $x=\frac{1}{2}\left(p_{2} e^{q_{1}}+e^{-q_{1}}\right)$, $y=\frac{1}{2} \sqrt{\frac{c}{b}}\left(e^{-q_{1}}-p_{2} e^{q_{1}}\right), z=-\sqrt{\frac{c}{b}} \cdot p_{1}-\frac{a}{b}$. It follows that $\varphi$ is a surjective submersion, the equations (2.4) are mapped onto the equations (1.1) and the canonical structure $\{., .\}_{\omega}$ is mapped onto the Poisson structure $\widetilde{\Pi}_{1}$, as required. We also remark that $H_{2}=\tilde{H}_{2}^{+}$and $C_{2}=\frac{c}{2} p_{2}$.

Theorem 2.6. Let $a, b, c, d, k \in \mathbb{R}$ such that $b d-a c=0$. If $b c<0$, then the Hamilton-Poisson mechanical system $\left(\mathbb{R}^{3}, \widetilde{\Pi}_{1}, H_{2}\right)$ has a full symplectic realization $\left(\mathbb{R}^{4}, \omega, \tilde{H}_{2}^{-}\right)$where $\omega=d p_{1} \wedge$ $d q_{1}+d p_{2} \wedge d q_{2}$ and $\tilde{H}_{2}^{-}=\frac{k}{2} p_{2}^{2} \cos ^{2} q_{1}-\frac{c}{2} p_{1}^{2}$.

Proof. The corresponding Hamilton's equations are

$$
\left\{\begin{array}{l}
\dot{q}_{1}=-c p_{1} \\
\dot{q}_{2}=k p_{2} \cos ^{2} q_{1} \\
\dot{p}_{1}=k p_{2}^{2} \sin q_{1} \cos q_{1} \\
\dot{p}_{2}=0
\end{array}\right.
$$

We define the application $\varphi: \mathbb{R}^{4} \rightarrow \mathbb{R}^{3}$ by $\varphi\left(q_{1}, q_{2}, p_{1}, p_{2}\right)=(x, y, z)$ where $x=p_{2} \cos q_{1}, y=$ $\sqrt{-\frac{c}{b}} \cdot p_{2} \sin q_{1}, z=-\sqrt{-\frac{c}{b}} \cdot p_{1}-\frac{a}{b}$. It follows that $\varphi$ is a surjective submersion, the equations (2.5) are mapped onto the equations (1.1) and the canonical structure $\{., .\}_{\omega}$ is mapped onto the Poisson structure $\widetilde{\Pi}_{1}$, as required. We also remark that $H_{2}=\tilde{H}_{2}^{-}$and $C_{2}=\frac{c}{2} p_{2}^{2}$.

We conclude this section noting that these Hamiltonian formulations are not of the type studied in [9].

\section{Symmetries}

In this section some types of symmetries are studied.

We recall that for a system $\dot{x}=f(x)$, where $f: M \rightarrow T M$, and $M$ is a smooth manifold of finite dimension, a vector field $\mathbf{X}$ is called:

- a symmetry if $\frac{\partial \mathbf{X}}{\partial t}+\left[\mathbf{X}, \mathbf{X}_{f}\right]=0$ where $\mathbf{X}_{f}$ is the vector field defined by the system;

- a Lie-point symmetry if its first prolongation transforms solutions of the system into other solutions; 
- a conformal symmetry if the Lie derivative along $\mathbf{X}$ satisfies $L_{\mathbf{X}} \pi=\lambda \pi$ and $L_{\mathbf{X}} H=\nu H$, for some scalars $\lambda, v$ where the Poisson tensor $\pi$ and the Hamiltonian $H$ give the Hamilton-Poisson realization of the system;

- a master symmetry if $\left[\left[\mathbf{X}, \mathbf{X}_{f}\right], \mathbf{X}_{f}\right]=0$, but $\left[\mathbf{X}, \mathbf{X}_{f}\right] \neq 0$.

For Lagrange's equations $\frac{d}{d t}\left(\frac{\partial L}{\partial \dot{q}_{i}}\right)-\frac{\partial L}{\partial q_{i}}=0, i=\overline{1,2}$ generated by the Lagrangian $L$, a vector field $\vec{v}=\xi\left(q_{1}, q_{2}, t\right) \frac{\partial}{\partial t}+\eta_{1}\left(q_{1}, q_{2}, t\right) \frac{\partial}{\partial q_{1}}+\eta_{2}\left(q_{1}, q_{2}, t\right) \frac{\partial}{\partial q_{2}}$ is

- a variational symmetry if $\mathrm{pr}^{(1)}(\vec{v})(L)+L \frac{d \xi}{d t}=0$ where $p r^{(1)}(\vec{v})=\vec{v}+\left(\dot{\eta}_{1}-\dot{\xi} \dot{q}_{1}\right) \frac{\partial}{\partial \dot{q}_{1}}+\left(\dot{\eta}_{2}-\dot{\xi} \dot{q}_{2}\right) \frac{\partial}{\partial \dot{q}_{2}} ;$

- a Lie-point symmetry if the action of its second prolongation $\operatorname{pr}^{(2)}(\vec{v})$ on Lagrange's equations vanishes where $p r^{(2)}(\vec{v})=p r^{(1)}(\vec{v})+\left(\ddot{\eta}_{1}-\ddot{\xi}_{\dot{q}_{1}}-2 \dot{\xi}_{\ddot{q}_{1}}\right) \frac{\partial}{\partial \ddot{q}_{1}}+\left(\ddot{\eta}_{2}-\ddot{\xi}_{\dot{q}_{2}}-2 \dot{\xi} \ddot{q}_{2}\right) \frac{\partial}{\partial \ddot{q}_{2}}$.

Following [8], our first result provides some Lie point symmetries and a conformal symmetry of the system (1.1).

Proposition 3.1. (i) The vector field $\mathbf{X}_{1}=-t \frac{\partial}{\partial t}+x \frac{\partial}{\partial x}+y \frac{\partial}{\partial y}+\left(z+\frac{a}{b}\right) \frac{\partial}{\partial z}$ is a Lie point symmetry of the system (1.1) in the case $b d-a c=0, b c \neq 0$.

(ii) The vector field $\mathbf{X}_{2}=-t \frac{\partial}{\partial t}+x \frac{\partial}{\partial x}+2 y \frac{\partial}{\partial y}+2\left(z+\frac{d}{c}\right) \frac{\partial}{\partial z}$ is a Lie point symmetry of the system (1.1) in the case $b=0, a c \neq 0$.

Moreover, $\mathbf{X}_{1}$ is a conformal symmetry.

Proof. If the vector $\mathbf{v}=\tau(t, x, y, z) \frac{\partial}{\partial t}+A_{1}(t, x, y, z) \frac{\partial}{\partial x}+A_{2}(t, x, y, z) \frac{\partial}{\partial y}+A_{3}(t, x, y, z) \frac{\partial}{\partial z}$ is a Lie point symmetry, then its first prolongation $p r^{(1)}(\mathbf{v})=\mathbf{v}+\left(\dot{A}_{1}-\dot{\tau} \dot{x}\right) \frac{\partial}{\partial \dot{x}}+\left(\dot{A}_{2}-\dot{\tau} \dot{y}\right) \frac{\partial}{\partial \dot{y}}+\left(\dot{A}_{3}-\dot{\tau} \dot{z}\right) \frac{\partial}{\partial \dot{z}}$ applied to our system implies

$$
\left\{\begin{array}{l}
\dot{A}_{1}-\dot{x} \dot{\tau}-(a+b z) A_{2}-b y A_{3}=0 \\
\dot{A}_{2}-\dot{y} \dot{\tau}-(d+c z) A_{1}-c x A_{3}=0 \\
\dot{A_{3}}-\dot{z} \dot{\tau}+k y A_{1}+k x A_{2}=0 .
\end{array}\right.
$$

One solution of the above system is the vector $\mathbf{X}_{1}$ in the case (i) and the vector $\mathbf{X}_{2}$ in the case (ii), i.e. $\mathbf{X}_{1}$ and $\mathbf{X}_{2}$ are Lie point symmetries.

One can easily check that $L_{\mathbf{X}_{1}} \widetilde{\Pi}_{1}=-\widetilde{\Pi}_{1}, L_{\mathbf{X}_{1}} \widetilde{\Pi}_{2}=-\widetilde{\Pi}_{2}, L_{\mathbf{X}_{1}} H_{2}=2 H_{2}, L_{\mathbf{X}_{1}} C_{2}=2 C_{2}$, whence $\mathbf{X}_{1}$ is a conformal symmetry.

The following result provides a master symmetry of our considered system.

Proposition 3.2. $i)$ The vector field $\vec{X}_{1}=x \frac{\partial}{\partial x}+y \frac{\partial}{\partial y}+\left(z+\frac{a}{b}\right) \frac{\partial}{\partial z}$ is a master symmetry of the system (1.1) in the case $b d-a c=0, b c \neq 0$.

(ii) The vector field $\vec{X}_{2}=x \frac{\partial}{\partial x}+2 y \frac{\partial}{\partial y}+2\left(z+\frac{d}{c}\right) \frac{\partial}{\partial z}$ is a master symmetry of the system (1.1) in the case $b=0, a c \neq 0$.

In the following, using symplectic realizations of the system (1.1), the symmetries of the EulerLagrange equations are presented.

Our study begins with the case $b d-a c=0, b c>0$. From Hamilton's equations (2.4) we obtain by differentiation:

$$
\left\{\begin{array}{l}
\ddot{q}_{2}-2 \dot{q}_{1} \dot{q}_{2}+\frac{k}{2} \dot{q}_{1}=0 \\
\ddot{q}_{1}-2 c e^{-2 q_{1}} \dot{q}_{2}+\frac{4 c}{k} e^{-2 q_{1}} \dot{q}_{2}^{2}=0 .
\end{array}\right.
$$


These are also the Euler-Lagrange equations generated by $L=\frac{1}{2 c} \dot{q}_{1}^{2}+\frac{2}{k} e^{-2 q_{1}} \dot{q}_{2}^{2}-e^{-2 q_{1}} \dot{q}_{2}$. The condition for the vector field $\vec{v}=\xi\left(q_{1}, q_{2}, t\right) \frac{\partial}{\partial t}+\eta_{1}\left(q_{1}, q_{2}, t\right) \frac{\partial}{\partial q_{1}}+\eta_{2}\left(q_{1}, q_{2}, t\right) \frac{\partial}{\partial q_{2}}$ to be a Lie Point symmetry for the Euler-Lagrange equations (3.1) leads to:

$\ddot{\eta}_{2}-\dot{q}_{2} \ddot{\xi}+\left(\frac{k}{2}-2 \dot{q}_{2}\right) \dot{\eta}_{1}-2 \dot{q}_{1} \dot{\eta}_{2}+\left(4 \dot{q}_{1} \dot{q}_{2}-\frac{k}{2} \dot{q}_{1}-2 \ddot{q}_{2}\right) \dot{\xi}=0$,

$\ddot{\eta}_{1}-\dot{q}_{1} \ddot{\xi}+\left(\frac{8 c}{k} \dot{q}_{2}-2 c\right) e^{-2 q_{1}} \dot{\eta}_{2}+\left(2 c e^{-2 q_{1}} \dot{q}_{2}-\frac{8 c}{k} e^{-2 q_{1}} \dot{q}_{2}^{2}-2 \ddot{q}_{1}\right) \dot{\xi}+\left(4 c \dot{q}_{2}-\frac{8 c}{k} \dot{q}_{2}^{2}\right) e^{-2 q_{1}} \eta_{1}=0$.

Taking into account the chain rule for the computations of $\dot{\xi}, \ddot{\xi}, \dot{\eta}_{1}, \ddot{\eta}_{1}, \dot{\eta}_{2}$ and $\ddot{\eta}_{2}$, and using the equations (3.1), the above equations become two equations in $t, q_{1}, q_{2}, \dot{q}_{1}, \dot{q}_{2}$, that are all independent. Then, these equations must be satisfied identically in $t, q_{1}, q_{2}, \dot{q}_{1}, \dot{q}_{2}$. It follows $\xi_{q_{1}}=$ $0, \xi_{q_{2}}=0, \eta_{1, q_{1}}=0, \xi_{t t}=0, \eta_{1, q_{2} q_{2}}=0, \eta_{2, q_{1} q_{2}}=0$ and

$$
\begin{aligned}
& \eta_{2, q_{1} q_{1}}-2 \eta_{2, q_{1}}=0, \\
& 2 \eta_{2, t t}+k \eta_{1, t}=0, \\
& \eta_{2, q_{2} q_{2}}-2 \eta_{1, q_{2}}-\frac{4 c}{k} e^{-2 q_{1}} \eta_{2, q_{1}}=0, \\
& 4 \eta_{2, t q_{1}}-4 \eta_{2, t}+k \xi_{t}-k \eta_{2, q_{2}}=0, \\
& k \eta_{1, q_{2}}-4 \eta_{1, t}+4 \eta_{2, t q_{2}}+4 c e^{-2 q_{1}} \eta_{2, q_{1}}=0, \\
& \eta_{1}-\eta_{2, q_{2}}=0 \\
& \eta_{1, t t}-2 c e^{-2 q_{1}} \eta_{2, t}=0, \\
& \eta_{1, q_{2}}+\frac{4 c}{k} e^{-2 q_{1}} \eta_{2, q_{1}}=0, \\
& \eta_{1, t q_{2}}+\frac{4 c}{k} e^{-2 q_{1}} \eta_{2, t}+2 c e^{-2 q_{1}} \eta_{1}-c e^{-2 q_{1}} \eta_{2, q_{2}}-c e^{-2 q_{1}} \xi_{t}=0 .
\end{aligned}
$$

Using (3.3), (3.5), (3.7) it results $\eta_{1, t}=0, \eta_{2, t t}=0, \eta_{2, t q_{2}}=0$. By (3.8) one gets $\eta_{2, t}=0$, whence $\eta_{1}=\xi_{t}$ from (3.7) and (3.10). Therefore $\eta_{2, q_{1}}=0$ by (3.9), hence $\eta_{2, q_{2} q_{2}}=0$ from (3.4). Taking into account the above results we obtain $\xi=\alpha t+\beta, \eta_{1}=\alpha, \eta_{2}=\alpha q_{2}+\gamma, \alpha, \beta, \gamma \in \mathbb{R}$.

Now, we can conclude the following result.

Theorem 3.1. Let $a, b, c, d, k \in \mathbb{R}$ such that $b d-a c=0$ and $b c>0$. The symmetries of the equations (3.1) are given by $\vec{v}=(\alpha t+\beta) \frac{\partial}{\partial t}+\alpha \frac{\partial}{\partial q_{1}}+\left(\alpha q_{2}+\gamma\right) \frac{\partial}{\partial q_{2}}$ where $\alpha, \beta, \gamma \in \mathbb{R}$.

Remark 3.1. (i) For $\alpha=\gamma=0$ and $\beta \neq 0$, we have $\overrightarrow{v_{1}}=\beta \frac{\partial}{\partial t}$ that represents the time translation symmetry which generates the conservation of energy $\widetilde{H}_{2}^{+}$.

(ii) For $\alpha=\beta=0$ and $\gamma \neq 0$, we have $\overrightarrow{v_{2}}=\gamma \frac{\partial}{\partial q_{2}}$ that represents a translation in the cyclic $q_{2}$ direction which is related to the conservation of $p_{2}$.

Moreover $\overrightarrow{v_{1}}$ and $\overrightarrow{v_{2}}$ are variational symmetries.

Remark 3.2. The 3-dimensional Lie algebra corresponding to the symmetries of the equations (3.1) endowed with the standard Lie bracket vector fields is generated by the base $\left\{\vec{u}_{1}, \vec{u}_{2}, \vec{u}_{3}\right\}$ where $\vec{u}_{1}=-t \cdot \frac{\partial}{\partial t}-\frac{\partial}{\partial q_{1}}-q_{2} \cdot \frac{\partial}{\partial q_{2}}, \vec{u}_{2}=\frac{\partial}{\partial t}, \vec{u}_{3}=\frac{\partial}{\partial q_{2}}$. The following relations $\left[\vec{u}_{1}, \vec{u}_{2}\right]=$ $\vec{u}_{2},\left[\vec{u}_{1}, \vec{u}_{3}\right]=\vec{u}_{3},\left[\vec{u}_{2}, \vec{u}_{3}\right]=\overrightarrow{0}$, hold. Therefore this Lie algebra is of type $\mathrm{V}$ in Bianchi's classification [1].

In the same manner the symmetries in the other cases are obtained.

In the case $b d-a c=0, b c<0$, from Hamilton's equations (2.5) we obtain:

$$
\left\{\begin{array}{l}
\ddot{q}_{2} \cos q_{1}+2 \dot{q}_{1} \dot{q}_{2} \sin q_{1}=0 \\
k \ddot{q}_{1} \cos ^{3} q_{1}+c \dot{q}_{2}^{2} \sin q_{1}=0 .
\end{array}\right.
$$


These are also the Euler-Lagrange equations generated by the Lagrangian $L=-\frac{1}{2 c} \dot{q}_{1}^{2}+\frac{1}{2 k \cos ^{2} q_{1}} \dot{q}_{2}^{2}$. Doing the same manipulation as before, we get that the symmetries of the equations (3.11) are given by $\vec{v}=\beta \frac{\partial}{\partial t}+\gamma \frac{\partial}{\partial q_{2}}, \beta, \gamma \in \mathbb{R}$, and again $\overrightarrow{v_{1}}$ and $\overrightarrow{v_{2}}$ are obtained.

In the case $b d-a c \neq 0, b=0$, we obtain from Hamilton's equations (2.2):

$$
\left\{\begin{array}{l}
\ddot{q}_{1}-a c k \dot{q}_{2} q_{1}-a d q_{1}=0 \\
a \ddot{q}_{2}+\dot{q}_{1} q_{1}=0,
\end{array}\right.
$$

which are also the Euler-Lagrange equations generated by $L=\frac{1}{2 a} \dot{q}_{1}^{2}+\frac{a c k}{2} \dot{q}_{2}^{2}+\frac{c k}{2} \dot{q}_{2} q_{1}^{2}+\frac{d}{2} q_{1}^{2}$.

In this case we have the following result.

Theorem 3.2. Let $a, b, c, d, k \in \mathbb{R}$ such that $b d-a c \neq 0$ and $b=0$. The symmetries of the equations (3.12) are given by $\vec{v}=(\alpha t+\beta) \frac{\partial}{\partial t}-\alpha q_{1} \frac{\partial}{\partial q_{1}}+\left(-\alpha q_{2}-\frac{2 d}{c k} \alpha t+\gamma\right) \frac{\partial}{\partial q_{2}}$ where $\alpha, \beta, \gamma \in \mathbb{R}$.

Remark 3.3. (i) For $\alpha=\gamma=0$ and $\beta \neq 0$, we have $\overrightarrow{v_{1}}=\beta \frac{\partial}{\partial t}$ that represents the time translation symmetry which generates the conservation of energy $\widetilde{H}_{1}$.

(ii) For $\alpha=\beta=0$ and $\gamma \neq 0$, we have $\overrightarrow{v_{2}}=\gamma \frac{\partial}{\partial q_{2}}$ that represents a translation in the cyclic $q_{2}$ direction which is related to the conservation of $p_{2}$.

Moreover $\overrightarrow{v_{1}}$ and $\overrightarrow{v_{2}}$ are variational symmetries.

Remark 3.4. The 3-dimensional Lie algebra corresponding to the symmetries of the equations (3.12) endowed with the standard Lie bracket vector fields is generated by the base $\left\{\vec{u}_{1}, \vec{u}_{2}, \vec{u}_{3}\right\}$ where $\vec{u}_{1}=-t \cdot \frac{\partial}{\partial t}+q_{1} \cdot \frac{\partial}{\partial q_{1}}+\left(q_{2}+\frac{2 d}{c k} t\right) \cdot \frac{\partial}{\partial q_{2}}, \vec{u}_{2}=\frac{\partial}{\partial t}, \vec{u}_{3}=\frac{\partial}{\partial q_{2}}$. We have the following bracket relations: $\left[\vec{u}_{1}, \vec{u}_{2}\right]=\vec{u}_{2}-\frac{2 d}{c k} \vec{u}_{3},\left[\vec{u}_{1}, \vec{u}_{3}\right]=-\vec{u}_{3},\left[\vec{u}_{2}, \vec{u}_{3}\right]=\overrightarrow{0}$.

In the case $b d-a c \neq 0, b \neq 0$, we obtain from the equations (2.2) the Euler-Lagrange equations

$$
\left(a+b k \dot{q}_{2}\right)^{2} \ddot{q}_{1}+b k \dot{q}_{1}^{2} q_{1}-\left(d q_{1}+c k \dot{q}_{2} q_{1}\right)\left(a+b k \dot{q}_{2}\right)^{3}=0, \quad\left(a+b k \dot{q}_{2}\right) \ddot{q}_{2}+\dot{q}_{1} q_{1}=0,
$$

generated by the Lagrangian $L=\frac{\dot{q}_{1}^{2}}{2 a+2 b k \dot{q}_{2}}-\frac{k}{2}(b d-a c) \dot{q}_{2}^{2}+\frac{c k}{2} \dot{q}_{2} q_{1}^{2}+\frac{d}{2} q_{1}^{2}$ and again as before we obtain $\vec{v}=\beta \frac{\partial}{\partial t}+\gamma \frac{\partial}{\partial q_{2}}, \beta, \gamma \in \mathbb{R}$.

\section{Conclusions}

In this paper a unitary presentation of the symmetries of a class of three-dimensional dynamical systems is given. In order to obtain this presentation, Hamiltonian structures, symplectic realizations and Lagrangian formulations are given.

The well-known dynamical systems as the Euler equations for a free rigid body, the equations of the rigid body with a free spinning rotor, the real Maxwell-Bloch equations, a particular case of Rikitake system, a particular case of Rabinovich system, a completely integrable case of the Lorenz system, a particular case of May-Leonard system and a particular case of Chen-Lee system, belong to the considered class.

Our unitary presentation allows the study of these classes of dynamical systems from other points of view, e.g. stability problems, existence of periodic orbits, homoclinic and heteroclinic orbits.

\section{Acknowledgements}

We would like to thank the referees very much for their valuable comments and suggestions. 


\section{References}

[1] L. Bianchi, Sugli spazii a tre dimensioni che ammettono un gruppo continuo di movimenti, Soc. Ital. Sci. Mem. Mat., Ser. IIIa 11 (1897) 267.

[2] G.W. Bluman, S. Kumei, Symmetries and Differential Equations, Appl. Math. Sci. 81 (Springer Verlag, New York, 1989).

[3] I. Caşu, Symmetries of the Maxwell-Bloch equations with the rotating wave approximation, Regular and Chaotic Dynamics 19, No. 5 (2014) 548-555.

[4] H.-K. Chen, C.-I. Lee, Anti-control of chaos in rigid body motion, Chaos, Solitons and Fractals 21 (4) (2004) 957-965.

[5] R.H. Cushman, L. Bates, Global Aspects of Classical Integrable Systems (Birkhäuser, Basel, 1997).

[6] P.A. Damianou, Master symmetries and R-matrices for the Toda Lattice, Lett. in Math. Phys. 20 (1990) 101-112.

[7] P.A. Damianou, Multiple hamiltonian structures for Toda systems of type A-B-C, Regular and Chaotic Dynamics 5 (1) (2000) 17-32.

[8] P.A. Damianou, P.G. Paschali, Symmetries of Maxwell-Bloch equations, J. Nonlinear Math. Phys. 2 N 3-4 (1995) 269-278.

[9] P.A. Damianou, C. Sophocleous, Symmetries of Hamiltonian systems with two degrees of freedom, J. Math. Phys. 40 (1999) 210-235.

[10] P.A. Damianou, C. Sophocleous, Symmetry group classification of three-dimensional Hamiltonian systems, Appl. Math. Lett. 13 (2) (2000) 63-70.

[11] D. David, D.D. Holm, Multiple Lie-Poisson Structures, Reductions, and Geometric Phases for the Maxwell-Bloch Travelling Wave Equations, J. Nonlinear Sci. 2 (1992) 241-262.

[12] A.S. Fokas, B. Fuchssteiner, The Hierarchy of the Benjamin-Ono equations, Phys. Lett. 86 A (1981) 341-345.

[13] H. Gümral, Y. Nutku, Poisson structure of dynamical systems with three degrees of freedom, J. Math. Phys. 34 (1993) p.5691, 1-33.

[14] P.S. Krishnaprasad, Lie-Poisson structures, dual-spin spacecraft and asymptotic stability, Nonl. An. Th. Meth. and Appl. 9 (1985) 1011-1035.

[15] C. Lăzureanu, T. Bînzar, Symplectic realizations and symmetries of a Lotka-Volterra type system, Regul. Chaotic Dyn. 18, No.3 (2013) 203-213.

[16] P. Libermann, C.-M. Marle, Symplectic Geometry and Analytical Mechanics (D. Reidel, Dordrecht, 1987).

[17] S. Lie, Vorlesungen über differentialgleichungen mit bekannten infinitesimalen transformationen (Teubner, Leipzig, 1891).

[18] J. Marsden, T.S. Raţiu, Introduction to Mechanics and Symmetry, 2nd Ed. Text and Appl. Math. 17 (Springer, Berlin, 1999).

[19] E. Noether, Invariante Variationsprobleme. Nachr. v. d. Ges. d. Wiss. zu Gottingen, Math. Phys. Kl (1918), 235-257; English translation, Transp. Th. Stat. Phys. 1 (1971) 186-207.

[20] P.J. Olver, Applications of Lie groups to Differential Equations (Springer Verlag, New York, 1986).

[21] A.S. Pikovskii, M.I. Rabinovich, Stochastic behavior of dissipative systems, Soc. Sci. Rev. C, Math. Phys. Rev. 2 (1981) 165-208.

[22] T. Rikitake, Oscillations of a system of disk dynamos, Proc. Cambridge Philos. Soc. 54 (1958) 89-105. 